\title{
INTERLACING THEOREM FOR THE LAPLACIAN SPECTRUM OF A GRAPH
}

\author{
MIRJANA LAZIĆ
}

\begin{abstract}
It is well known that the Interlacing theorem for the Laplacian spectrum of a finite graph and its induced subgraphs is not true in a general case. In this paper we completely describe all simple finite graphs for which this theorem is true. Besides, we prove a variant of the Interlacing theorem for Laplacian spectrum and induced subgraphs of a graph which is true in general case.
\end{abstract}

\section{INTRODUCTION}

First we repeat in short some elementary facts about the Laplacian spectrum of a finite graph which we shall use in the sequel.

Let $G$ be a simple graph on $n$ vertices and the vertex set $V(G)=\left\{v_{1}, \ldots, v_{n}\right\}$. Next, let $A(G)=\left[a_{i j}\right]$ be its $(0,1)$ adjacency matrix, and $D(G)=\operatorname{diag}\left(d_{1}, \ldots, d_{n}\right)$ be the diagonal matrix with vertex degrees $d_{1}, \ldots, d_{n}$ of its vertices $v_{1}, \ldots, v_{n}$. Then $L(G)=D(G)-A(G)$ is called the Laplacian matrix of the graph $G$. It is symmetric, singular and positively definite. Its eigenvalues are all real and nonnegative and form the Laplacian spectrum $\sigma_{L}(G)=\left\{\lambda_{1}, \ldots, \lambda_{n}\right\}$ of the graph $G$. We shall always assume that $\lambda_{1} \geq \lambda_{2} \geq \cdots \geq \lambda_{n}$. It is well known that $\lambda_{n}=0$ and the multiplicity of 0 equals to the number of (connected) components of $G$. Hence, $\lambda_{k}(G)=0$ for some $k=1, \ldots, n$ if and only if $G$ has at least $n-k+1$ components.

Theorem A. If $H$ is a (not necessary induced) subgraph of a finite graph $G$ then

$$
\lambda_{k}(H) \leq \lambda_{k}(G) \quad(k=1, \ldots,|H|) .
$$

Next, let $G_{1}=\left(V\left(G_{1}\right), E\left(G_{1}\right)\right), \ldots, G_{m}=\left(V\left(G_{m}\right), E\left(G_{m}\right)\right)(m \geq 2)$ be finite graphs with mutually disjoint sets of vertices $V\left(G_{1}\right), \ldots, V\left(G_{m}\right)$. Then the direct sum $G=G_{1}+\cdots+G_{m}$ of these graphs is defined by $V(G)=V\left(G_{1}\right) \cup \cdots \cup V\left(G_{m}\right)$ and $E(G)=E\left(G_{1}\right) \cup \cdots \cup E\left(G_{m}\right)$.

Theorem B. If $G=G_{1}+\cdots+G_{m}$ is the direct sum of graphs $G_{1}, \ldots, G_{m}$, then

$$
\sigma_{L}\left(G_{1}+\cdots+G_{m}\right)=\sigma_{L}\left(G_{1}\right) \cup \cdots \cup \sigma_{L}\left(G_{m}\right)
$$

2000 Mathematics Subject Classification. Primary: 05C50.

Key words and phrases. Simple graphs, Laplacian spectrum. 
including the multiplicities too.

Theorem C. If $\bar{G}$ is the complementary graph of a graph $G$, then

$$
\lambda_{k}(\bar{G})=n-\lambda_{n-k}(G) \quad(k=1, \ldots, n-1) .
$$

If $G$ is a graph and $H$ is any its induced subgraph, we shall denote it by $H \subseteq G$. The void graph on $n$ vertices (without any edge) is denoted by $E_{n}$, the complete graph on $n$ vertices is denoted by $K_{n}$, and the star on $n$ vertices is denoted by $K_{1, n-1}$. The graph $K_{2}+\cdots+K_{2}\left(p\right.$ copies of the graph $\left.K_{2}\right)$ is denoted simply by $p K_{2}$.

\section{MAin Results}

By analogy to the known Interlacing theorem for the ordinary spectrum of a finite graph, we formulate a possible variant of the Interlacing theorem for the Laplacian spectrum of a graph. We shall call it "L.I.T." in short (the "Laplacian Interlacing Theorem").

L.I.T. If $G$ is a finite graph of order $n(n \in N)$, then for every its induced subgraph $H$ of order $m(m<n)$, it holds

$$
\lambda_{n-m+k}(G) \leq \lambda_{k}(H) \leq \lambda_{k}(G) \quad(k=1, \ldots, m) .
$$

Note that by Theorem A the right-side of (1) is always true, even for an arbitrary subgraph $H$ of $G$. Hence, the only interesting part of L.I.T. are in fact the inequalities

$$
\lambda_{k}(H) \geq \lambda_{n-m+k}(G) \quad(k=1, \ldots, m) .
$$

Unfortunately, such a general theorem is, as is well known, not true in the general case. There are many counter-examples, and we notice only one.

Let $G=K_{1, n}(n \geq 2)$ be the star with $n$ rays, and $H$ be the induced subgraph $E_{n} \subseteq G$ obtained by removal the central vertex of $G$. Then

$$
\sigma_{L}(G)=\{n+1, \underbrace{1, \ldots, 1}_{n-1}, 0\}, \quad \sigma_{L}(H)=\{\underbrace{0, \ldots, 0}_{n}\},
$$

so that (2) obviously fails, because $\lambda_{1}(H)=0<\lambda_{2}(G)=1$.

Therefore, we pose the following question:

Find all finite graphs $G$ such that L.I.T. holds for $G$.

The next theorem completely resolves this question.

Theorem 1. A graph $G$ satisfies L.I.T. if and only if $G=G(p, q)=p K_{2}+E_{q}$ for some integers $p, q \geq 0(p+q \geq 1)$.

Proof. First suppose that $G$ is an arbitrary graph of the form $G(p, q)(p+q \geq 1)$. The Laplacian spectrum of $G(p, q)$ reads:

$$
\sigma_{L}(G(p, q))=\{\underbrace{2, \ldots, 2}_{p}, \underbrace{0,0, \ldots, 0}_{p+q}\} .
$$

If $H$ is any proper induced subgraph of $G$, then it is also of the form $G\left(p_{0}, q_{0}\right)$ $\left(p_{0}+q_{0} \geq 1\right)$, where obviously $p_{0} \leq p$. Since the number of components of $G(p, q)$ 
is $p+q$, and a removal of any number of vertices of the graph $G(p, q)$ together with the corresponding edges does not increase the number of components, we conclude that $p+q \geq p_{0}+q_{0}$. Next, we have that

$$
\sigma_{L}(H)=\{\underbrace{2, \ldots, 2}_{p_{0}}, \underbrace{0, \ldots, 0}_{p_{0}+q_{0}}\}
$$

so that obviously

$$
\lambda_{k}(H)=2 \geq \lambda_{n-m+k}(G) \quad\left(k=1, \ldots, p_{0}\right) .
$$

Here $n=2 p+q, m=2 p_{0}+q_{0}<n$.

Further, we have that $\lambda_{k}(H)=0\left(k=p_{0}+1, \ldots, m\right)$, and $\lambda_{n-m+k}(G)=0$ $\left(k=p_{0}+1, \ldots, m\right)$ since $n-m+k>n-m+p_{0} \geq p$, because $p+q \geq p_{0}+q_{0}$, as we have already said.

Hence, the inequalities (2) hold for every $k=1, \ldots, m$.

Conversely, let $G$ satisfies L.I.T., and let $G_{1}, \ldots, G_{r}(r \geq 1)$ be the (connected) components of $G$. We first wish to prove that each component $G_{i}$ is a complete graph $(i=1, \ldots, r)$.

On the contrary, suppose that for instance $G_{1}$ is not complete. Let $v_{1}^{\prime}, v_{1}^{\prime \prime}$ be two nonadjacent vertices in $G_{1}$, and $v_{2} \in V\left(G_{2}\right), \ldots, v_{r} \in V\left(G_{r}\right)$ be arbitrary fixed vertices. Then $v_{1}^{\prime}, v_{1}^{\prime \prime}, v_{2}, \ldots, v_{r}$ form an induced subgraph $H \subseteq G$ which is void, so by (2) we find that

$$
\lambda_{1}(H)=0 \geq \lambda_{n-(r+1)+1}(G)=\lambda_{n-r}(G),
$$

thus $\lambda_{n-r}(G)=0$. But the last equality means that that $G$ has at least $r+$ 1 components, what is a contradiction. Hence, all components $G_{1}, \ldots, G_{r}$ are complete graphs. Without loss of generality we can assume that for some $p \geq 0$ $G_{1}=K_{n_{1}}, \ldots, G_{p}=K_{n_{p}}\left(n_{1}, \ldots, n_{p} \geq 2\right)$ and $G_{i}=K_{1}(i=p+1, \ldots, r)$, so that $G=K_{n_{1}}+\cdots+K_{n_{p}}+E_{q}(p+q=r \geq 1)$. We can also assume that $2 \leq n_{1} \leq n_{2} \leq \cdots \leq n_{p}$.

Next, we wish to prove that $n_{1}=2$. We obviously have that

$$
\sigma_{L}(G)=\{\underbrace{n_{p}, \ldots, n_{p}}_{n_{p}-1}, \ldots, \underbrace{n_{1}, \ldots, n_{1}}_{n_{1}-1}, \underbrace{0, \ldots, 0}_{p+q}\} .
$$

Suppose on the contrary that $n_{1} \geq 3$. Removing a vertex from the component $K_{n_{1}}$, we obtain an induced subgraph $H \subseteq G$, and

$$
\sigma_{L}(H)=\{\underbrace{n_{p}, \ldots, n_{p}}_{n_{p}-1}, \ldots, \underbrace{n_{1}-1, \ldots, n_{1}-1}_{n_{1}-2}, \underbrace{0, \ldots, 0}_{p+q}\} .
$$

Since $n_{1}-2 \geq 1$, by (2) we easily get a contradiction $n_{1}-1 \geq n_{1}$.

Therefore $n_{1}=2$. Continuing this reasoning, we consecutively find that $n_{2}=$ $2, \ldots n_{p}=2$, so that $G=G(p, q)=p K_{2}+E_{q}$ where $p+q \geq 1$. This completes the proof.

Finally, we formulate another variant of the Interlacing theorem which is more appropriate to the Laplacian spectrum of a graph, and is true in the general case. 
Theorem 2. If $G$ is a graph of order $n$ and $H$ is any its induced subgraph of order $m(m<n)$, then it holds:

$$
\lambda_{n-m+k}(G)-n+m \leq \lambda_{k}(H) \leq \lambda_{k}(G) \quad(k=1, \ldots, m) .
$$

Proof. We only need to prove the left inequalities in (3).

First, it is obviously true for $k=m$ because $\lambda_{n}(G)=\lambda_{m}(H)=0$ and $n>m$.

Next, assume that $k \leq m-1$. Denoting by $\bar{G}$ the complement of $G$ and by $\bar{H}$ the complement of $H$, we have that $\bar{H}$ is an induced subgraph of $\bar{G}$, so that

$$
\lambda_{k}(\bar{H}) \leq \lambda_{k}(\bar{G}) \quad(k=1, \ldots, m-1) .
$$

But since $\lambda_{k}(\bar{G})=n-\lambda_{n-k}(G)$ and $\lambda_{k}(\bar{H})=m-\lambda_{m-k}(G)(k=1, \ldots, m-1)$, we find that

$$
m-\lambda_{m-k}(H) \leq n-\lambda_{n-k}(G) \quad(k=1, \ldots, m-1) .
$$

Replacing $k$ with $m-k$, we get

$$
\lambda_{k}(H) \geq \lambda_{n-m+k}(G)-n+m \quad(k=1, \ldots, m-1),
$$

and finally

$$
\lambda_{k}(H) \geq \lambda_{n-m+k}(G)-n+m \quad(k=1, \ldots, m) .
$$

Obviously, the above inequalities have a sense only for values $k \leq m$ such that $\lambda_{n-m+k}(G) \geq n-m$.

Also notice that the previous proof can not be used if $H$ is an arbitrary subgraph of a graph $G$, since in this case $\bar{H}$ is not necessary a subgraph of the graph $\bar{G}$. Moreover, this statement is again not true for subgraphs of a graph in the general case.

\section{REFERENCES}

[1] D. M. Cvetković, M. Doob, H. Sachs, Spectra of graphs, Academic Press, New York, 1979.

[2] D. M. Cvetković, M. Doob, I. Gutman, A. Torgašev, Recent results in the Theory of graph Spectra, Ann. Discrete Math. 36(1988), North-Holland, Amsterdam, 1988.

[3] R. Grone, R. Merris, V. S. Sunders, The Laplacian spectrum of a graph, SIAM J. Matrix Anal. Appl. 11(1990), 218-238.

[4] R. Merris, Laplacian matrices of graphs, A survey, Linear Algebra and its Appl. 197, 198(1994), 143-176.

[5] B. Mohar, The Laplacian spectrum of graphs, Graph theory, Combinatorics and Appl. (ed. by Y. Alard, G. Chartrand, O.R. Ollerman, A.J.Schwenk), J. Willy Inc., New York, 1991; pp. 871-898. 
FACUlTy of ScIEnCE

Institute OF MATHEMATICS AND INFORMATICS

P.O.Box 60, Radoja Domanovića 12

34000 Kragujevad

Serbia And Montenegro

E-mail address: mmmvl@kg.ac.yu 